\title{
Cardiomiopatía periparto y tumor desmoide abdominal: presentación de un caso y revisión de la literatura
}

\author{
Janer Sepúlveda Agudelo*; Víctor Armando Pinto Barón**
}

Recibido: Abril 12 I 2000

Revisado: Abril 24 I 2000

Aceptado: Agosto 11 I 2000

\section{RESUMEN}

Presentamos el caso de una mujer de 27 años diagnosticado y manejado en el Hospital San Rafael de Tunja, Colombia con cardiomiopatía periparto asociado a una masa de origen abdominal que después fue clasificada por el estudio histopatológico como tumor desmoide abdominal. Ambas entidades son de una frecuencia poco común por lo cual se hace una revisión detallada de la literatura de estas patologías.

PALABRAS CLAVES: Cardiomiopatía periparto, postparto, diagnóstico, tumor desmoide.

\section{SUMMARY}

We present a case of a 27 years old woman diagnosed and treated in San Rafael Hospital Tunja Colombia with peripartum cardiomyopathy and tumor of the abdominal, witch was classified on pathological study how desmoid tumor. 80th entities are infrequent, we reviews the literature of these pathologies.

KEY WORDS: Peripartum cardiomyopathy, postpartum, diagnosis, desmoid tumor.

\section{Introducción}

La cardiomiopatía periparto es una forma poco usual de insuficiencia cardíaca congestiva de causa desconocida que puede tener consecuencias devastadoras sobre la evolución materna y fetal. La falla cardíaca asociada al embarazo, fue informada en 1849, pero hasta 1930 fue descrita como una nueva entidad.

La incidencia real de la cardiomiopatía periparto es desconocida (1), se estima un rango de 1: 3.000 al: 15.000 embarazos (1-2). Esta varía de acuerdo a la región geográfica que se estudie (3), es así como en Nigeria ha sido reportada hasta en un $1 \%$, relacionada con prácticas con excesivo consumo de sal en el postparto $(1,4-6)$.

El tumor desmoide fue originalmente descrito como un tumor de la pared abdominal en mujeres que recientemente han presentado un embarazo; pero este tumor puede presentarse en cualquier época de la vida y localizarse en otros sitios del cuerpo, su incidencia se estima en 2 a 4 casos por 100.000 (7).

\section{Caso clínico}

Paciente de 27 años de raza mestiza procedente del área rural de Miraflores (Boyacá) con antecedente de parto domiciliario 7 días previos al ingreso y posterior presencia de dolor abdominal de moderada intensidad, fiebre, malestar general y presencia de loquios fétidos, inician manejo con antibióticos para endometritis postparto y ante la no mejoría del cuadro clínico la remiten a esta institución (Hospital San Rafael de Tunja, Boyacá).

\section{Antecedentes sin importancia}

Al examen físico se encuentra una paciente en malas condiciones generales, con palidez mucocutánea marcada y taquicardia, cabeza y cuello normal.

Cardiopulmonar, ruidos cardíacos rítmicos, taquicárdicos sin sobreagregados.

Abdomen distendido, ruidos intestinales disminuidos con onda ascítica (+), útero no palpable a nivel abdominal, se palpaba masa infraumbilical de $+/-20 \times 15 \mathrm{~cm}$. Lisa adherida a planos profundos.

Tacto Vaginal, útero en anteverso flexión con buena involución, loquios no fétidos.

Extremidades, edema grado II de miembros inferiores. Se hace impresión diagnóstica inicial de carcinomatosis por cáncer de ovario.

Se realiza ecografía y tomografía abdomino-pélvica cuyos resultados son compatibles con tumor de ovario y carcinomatosis posiblemente de origen ovárico.

Se realizan 2 paracentesis, una de ellas guiada por ecografía, cuyos resultados son negativos para malignidad. Posteriormente la evolución de la paciente es tórpida, comienza a presentar disnea progresiva y al examen físico

MD. Especialistas en Ginecología Obstetricia. Universidad Industrial de Santander.

** Profesor Asociado. Departamento de Obstetricia y Ginecología. Universidad UPTC. Tunja. Hospital San Rafael de Tunja. 
se encontraban signos claros de falla cardíaca; por lo cual se inicia manejo respectivo.

La radiografía de tórax muestra cardiomegalia y derrame pleural, el electrocardiograma muestra taquicardia sin otros hallazgos patológicos; se revalúa caso clínico y se considera que puede tratarse de una cardiomiopatía periparto.

Se solicita ecocardiograma, el cual confirma el diagnóstico, se inicia digoxina y diuréticos con mejoría.

Se realiza nueva paracentesis, guiada por ecografía, la cual es reportada nuevamente negativa para malignidad.

Evolución posterior satisfactoria. En cuanto a los hallazgos a nivel abdominal en vista de 3 citologías previas negativas, se decide llevar la paciente a laparotomía exploratoria, donde se encuentra una masa pélvica de 20 x $15 \mathrm{~cm}$. que ocupa pelvis, hipogastrio y mesogástrio adherida firmemente a la pared abdominal anterior con adherencias laxas a peritoneo $\mathrm{y}$ asas intestinales, $\mathrm{y}$ adherida a fondo uterino de manera firme, de superficie lisa de aspecto de músculo liso con un grosor de 2-3 mm. la cual se extrae. Ovarios de aspecto normal, resultados de patología informan fibromatosis abdominal, (Tumor desmoide abdominal).

\section{Discusión}

La cardiomiopatía periparto representa menos del $1 \%$ de los problemas cardiovasculares asociados con el embarazo (8).

Los criterios clásicos para el diagnóstico según señalaron Demakis y cols. (1-3, 5,8-'9), son:

1. Aparición de insuficiencia cardíaca en el último mes de embarazo o en los cinco meses de postparto.

2. Ausencia de una causa determinable de la insuficiencia cardíaca

3. Ausencia de cardiopatía demostrable antes del último mes del embarazo.

El diagnóstico puede ser confirmado por un ecocardiograma al eliminar otras causas de disfunción miocárdica (4).

Esta entidad tiene mayor prevalencia en mujeres mayores de 30 años (1), en multíparas $(1,8)$, pacientes con embarazo gemelar (1, 3, 8-9) (Los embarazos gemelares pueden ocurrir cerca del $-10 \%$ de los casos, publicados de cardiomiopatía periparto); pacientes con historia de preeclampsia e hipertensión crónica (3-4, 6, 8-9).

La desnutrición y la anemia han sido reportadas como factores de riesgo $(1,5,8 i 9)$, al igual que abuso de cocaína, infección por entero virus y deficiencia de selenio (1).

Lampert y cols (1 O), encontraron el uso prolongado de terapia tocolítica mayor de cuatro semanas como factor de riesgo.

Otros autores han sugerido como etiología un proceso autoinmune $(2,5)$. La cardiomiopatía periparto no es ordinariamente familiar, pero casos hereditarios son ocasionalmente observados (11).

El cuadro clínico ocurre usualmente en el período post parto y solo en algunos pacientes antes del parto (5). Con mayor frecuencia en el segundo mes postparto (8).

Los síntomas y signos que se presentan son los de falla cardíaca y más específicamente los de falla cardíaca izquierda (8).
Los síntomas de presentación mas frecuentes son, disnea (se considera un hallazgo universal), tos, ortopnea, fatiga $y$ palpitaciones $(1,5,12)$. Puede ocurrir hemoptisis, dolor torácico y abdominal que no son específicos y ocurren en un $33 \%$ de los casos $(1,5)$.

Los signos más frecuentes son: aumento de la presión arterial (1) (puede estar normal o baja), cardiomegalia, taquicardia, estertores y arritmia; también puede haber soplos cardíacos, ingurgitación yugular ritmo de galope, S3, S4 y edemas $(1,5,10,12)$.

Cardiomegalia asintomática puede presentarse en un 4- 13\% (13); ocasionalmente puede haber embolización pulmonar; también se ha reportado como modo de presentación falla renal post parto (8), manifestaciones neurológicas, usualmente secundarias a fenómenos embólicos (8).

\section{Hallazgos paraclínicos}

Los exámenes que se deben solicitar incluyen electrocardiograma (EKG), radiografía de tórax y ecocardiograma Doppler (1). El EKG demuestra ritmo normal o taquicardia sinusal o raramente fibrilación auricular. El voltaje es normal o bajo, hay criterios de hipertrofia ventricular izquierda, puede haber anormalidades no específicas del segmento ST y de la onda T y ocasionalmente en la onda Q. El intervalo PR y el complejo QRS pueden estar prolongados sugiriendo defectos en la conducción intraventricular $(1-3,12)$.

La radiografía de tórax invariablemente muestra cardiomega1ia $(1-3,12)$ con derrame pleura1 bilateral ocasional $(1,3,12)$. En adición congestión venosa pulmonar e infiltrados bibasales son comúnmente vistos $(1,12)$.

El ecocardiograma usualmente muestra un ventrículo izquierdo dilatado con disminución en la función ventricular sistólica izquierda $(1,3,12,14)$. Trombos ventriculares pueden ser visualizados.

El papel de la biopsia endomiocárdica permanece controvertida y es de utilidad clínica solo cuando se realiza en el curso temprano de la enfermedad (1).

\section{Tratamiento}

\section{Anteparto}

La identificación del problema de la paciente en etapas tempranas del período anteparto proporciona tiempo para estructurar un plan de tratamiento eficaz, en el que debe considerarse vigilancia materno fetal anteparto, momento y día del parto, analgesia en el trabajo de parto, necesidad de profilaxis para endocarditis, anticoagulación, deseo de reproducción futura y la necesidad de cateterismo intraparto de la arteria pulmonar (5).

El tratamiento médico debe centrarse en el logro de la función hemodinámica materna óptima mediante la modificación de determinantes de la función miocárdica (Precarga, contractilidad y post carga) (5). La terapia es similar a otras formas de insuficiencia congestiva $(1-2,4)$.

El uso de diuréticos como la Furosemida debe realizarse cuando hay estertores pulmonares, mas hipoxemia materna $(1,5)$.

Cuando los síntomas de presentación son fatiga crónica, y tolerancia limitada al ejercicio se recomiendan 
digitálicos. Este medicamento es seguro durante el embarazo cuando se encuentra a nivel terapéutico $(1,5)$. También puede utilizarse Digitálicos para pacientes con fibrilación auricular (1, 5). Si no hay mejoría con esa droga puede ser benéfico disminuir la postcarga con hidralacina o captopril, se debe recordar que el captopril cruza la placenta y solo se debe utilizar si el beneficio materno es mayor que los riesgos fetales $(1,5)$, finalmente se debe hacer profilaxis con heparina para evitar complicaciones tromboembólicas $(1,4-5)$. Otra medida que se debe realizar es la restricción de sal a menos de 4grs por día y restricción de líquido a menos de 2 litros al día (1-4).

\section{Tratamiento Intraparto}

El trabajo del parto y el parto son períodos cruciales para la paciente con cardiomiopatía periparto; pues durante este período se ve sometida a una carga hemodinámica mayor relacionada con las cqntracciones uterinas, los anestésicos, los procedimientos quirúrgicos, la pérdida sanguínea y el tratamiento con líquidos intra venosos $(1,5)$.

Cuando se dispone de recursos de vigilancia hemodinámica invasiva se puede ofrecer un parto vaginal seguro. No es necesaria la profilaxis para endocarditis (5).

El tratamiento debe incluir oxígeno, diuréticos, inotrópicos y vasodilatores dependiendo del perfil hemodinámico basal (1, 4$5,12)$.

En el tratamiento intraparto se recomienda la dopamina a dosis de 2-5 microgramos/kilogramo/minuto; si no se logra una función ventricular satisfactoria con dopamina se debe añadir un segundo inotrópico como la Dobutamina $(2$ a 10 microgramo/kilogramo)(5).

Otras medidas son mantener la paciente en decúbito lateral, suministrar analgesia y de ser posible deberá abreviarse el segundo período del trabajo de parto; debe reservarse la cesárea para indicaciones obstétricas $(5,3)$.

\section{Tratamiento Postparto}

Debe realizarse vigilancia sistemática de la evolución por el cardiólogo; se debe valorarla necesidad de antico-agulación prolongada, ecocardiografía seriadas y el deseo potencial de embarazo futuros (5) .

Pacientes con insuficiencia cardíaca terminal, el trasplante cardíaco es el único tratamiento definitivo (5).

\section{Pronóstico}

Se reporta un rango de mortalidad de un 25 a $50 \%(1,4,15$ 16). La muerte usualmente ocurre por falla congestiva crónica progresiva, arritmia o complicaciones tromboembólicas $(1,12$, 15), se considera que el 50\% tienen una resolución espontánea generalmente en los 6 meses posteriores al diagnóstico $(2,9,12$, $15)$.

Una notable característica de lacardiomiopatía periparto es su tendencia a recurrir con subsecuentes embarazos (16-17). Se ha sugerido que el pronóstico para futuros embarazos está relacionada con el tamaño del corazón, pacientes que recobran normal dentro de 6 a 12 meses tienen una mortalidad de un 11-14\% en subsecuentes embarazos y en aquellas que persiste la cardiomiopatía tienen un índice de mortalidad del 40 al 80\% (16-17). Igualmente pacientes que recobran su función ventricular izquierda tienen una mayor supervivencia de 10 años, 85\% contra un $15 \%$ (18). En general se recomienda a estas mujeres no embarazarse nuevamente $(4,16)$.

\section{Tumor desmoide}

La biología y la historia natural de los tumores desmoides es desconocida; han sido clasificados histológicamente como tumores benignos. Varias teorías han sido sugeridas para la patogénesis de los tumores desmoides, incluyendo trauma, factores genéticos $\mathrm{y}$ estimulación estrogénica (20). Sin embargo estudios moleculares en el cromosoma $\mathrm{X}$ han confirmado que esas lesiones son el resultado de procesos clonales, estableciendo que los desmoides son neoplasias y no producto de reacción fibrosa inflamatoria intensa (19).

Son más frecuentes en la tercera y cuarta década de la vida (20). Ocurre generalmente en tres localizaciones (19, 21): Extraabdominal, pared abdominal, asas intestinales y mesenterio. La Extraabdominal principalmente en las extremidades, pero también se ha reportado un caso en laringe (20) y un caso en la vulva (22) ambos durante el embarazo. Los del mesenterio están asociados a menudo con poliposis adenomatosa familiar.

El $49 \%$ de los casos envuelve el abdomen (23), aunque otras series reportan con más frecuencia las extremidades (7).

La relación en frecuencia mujer a hombre es de 1.6: 1. Se ha visto con mayor relación en pacientes con síndrome de Gardner especialmente de localización retroperitoneal. Los desmoides extraabdominales son mas frecuentes en niños y los abdominales en el grupo de edad fértil (7); principalmente durante y después del embarazo (18).

La tomografía axial computarizada es el medio mas útil para el diagnóstico (21).

Aunque los desmoides no dan metástasis, para el manejo clínico de estos tumores se consideran como fibrosarcomas de bajo grado $(7,19)$. El tratamiento inicial es quirúrgico (19).

\section{Conclusión}

La cardiomiopatía periparto es una patología poco frecuente, la cual debe ser tenida en cuenta al descartar otras causas de insuficiencia cardíaca; su manejo tiene que realizarse por un grupo multidisciplinario y seguirse a estas pacientes hasta que recobren completamente su función ventricular izquierda. En lo posible se recomienda no embarazos subsecuentes, por las consecuencias que este puede traer para la salud materna y fetal.

El tumor desmoide es una entidad rara que debe siempre tenerse en cuenta como diagnóstico diferenciar en masas de la pared abdominal y así realizar un diagnóstico oportuno para un adecuado tratamiento. 


\section{BIBLIOGRAFIA}

1. Lampert MB, Lang RM. Peripartum cardiomyopathy. American Heart JournaI 1995; 130: 860-70.

2. Brown CS, Bertolet BD. Peripartum cardiomyopathy: A comprehensive review. Am J Obstet Gynecol 1998; 178: 409-14.

3. Homans DC. Current concepts peripartum cardiomyopathy. N Engl J Med 1985; 312: 1431-7.

4. Ridley DM, Smiley RM. La embarazada cardiópata. In: Suresh MS Clínicas de Anestesiología de Norte América. México: McGraw-Hill Interamericana, 1998; 2: 461-3.

5. Lee W, Cotton DB. Cardiomiopatía periparto conceptos actuales y tratamiento clínico. In: Pitkin RM, Gilstrap LC. Clínicas obstétricas y ginecológicas. México: McGraw-Hill Interamericana 1989; 1: 55-67.

6. Cunningham FG, Pritchard JA, Hankins DV, Anderson PL Lucas MI, Armstrong KF. Peripartum heart failure: Idiopathic cardiomyopathy or compounding cardiovascular events? Obstet Gynecol 1986; 67: 157.

7. De Vita VT, Hellman S, Rosenberg SA. Cancer y principIes and practice of oncology. 5a ed. Philadelphia: Lippincott Raven, 1997; 1747.

8. Veille JC. Peripartum cardiomyopathies: A review. Am $l$ Obstet Gyneacol 1984;148: 805-17.

9. Witlin AG, Mabie WC, Sibai BM. Peripartum cardiomyopathy: An ominous diagnosis. Am $l$ Obstet Gynecol 1997; 176: 182-8.

10. Lampert MB, Hibbard J, Weinert L, Briller J, Lindheimer M, Lang R. Peripartum heart failure associated with prolonged tocolytic therapy. Am J Obstet Gynecol 1993; 198: 493-5.

11. Shabetai R. Cardiac diseases. In: Creasy RK, Resnik R. Maternal fetal medicine principIes and practice. 3th ed. Philadelphia: W.B Saunders Company, 1994; 785-6.

12. Braunwald heart disease: A text book of cardiovascular medicine. 5a ed Philadelphia: W.B Saunders Company, 1997; 1850-2.

13. Dec GW, Fuster V. Idiopathic dilated cardiomyopathy. N Engl $l$ Med 1994; 331: 1564-75.
14. Melvin KR, Richardson PJ, Olsen EGJ, Daly K, Jackson G. Peipartum cardiomyopathy due to myocarditis. N Engl J Med 1982; 307:731-4

15. Lampert MB, Weinert L, Hibbard J, Korcarz C, Lindheimer M, Lang RM Contractile reserve in patients with peripartum cardiomyopathy and recovered left ventricular function. Am J Obstet Gynecol 1997; 176: $189-95$.

16. Clark SL. Cardiopatías en el embarazo. In: Cotton DB. Clínicas de Ginecología y Obstetricia. México, McGraw Hill: Interamericana, 1991; 2: 252-3.

17. Clark SL., Cotton DD. Hankins GDV, Phelan JP. Critical care obstetrics. 3th ed. United States of America, Braun-Brumfield, $1997 ; 301-2$.

18. Scott JR, Wagoner LE, Olsen SL. Pregnancy in heart transplant recipients Management and outcome. Obstet Gynecol 1993; 82: 324.

19. Lewis JJ, Boland PJ, Leung DHY y cols. The Enigma of Desmoid Tumors Annals of surgery. 1999; 229: 866-73

20. Gherman RB, Bowen E, Eggleston MK, Karakla D. Desmoid tumor of the larynx complicating pregnancy: A case report. Am J Obstet Gynecol 1999; 180: 1036-7.

21. Lambroza A, Tighe MK, DeCosse JJ, Dannenberg AJ. Disorders of the Rectus Abdominis Muscle and Sheath: A 22 year experience. Am J Gastroenterology 1995; 90: 1313-7.

22. Allen MV, Novotny DB. Desmoid tumor of the vulva associated with pregnancy. Arch Pathol Lab Med 1997; 121: 512-4. Medline

23. Reitamo JJ, Hayry p, Nykyri E, et al. The desmoid tumor: I. Incidence, sex-age-and anatomical distribution in the Finnish population. Am J Clin Pathol 1982; 77: 665-73. 